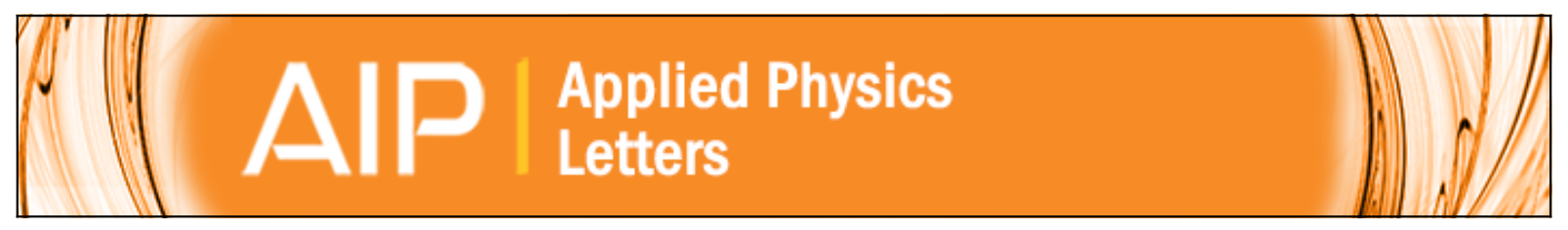

\title{
Near-field measurement of spectral anisotropy and optical absorption of isolated ZnO nanorod single-quantum-well structures
}

Takashi Yatsui, Motoichi Ohtsu, Jinkyoung Yoo, Sung Jin An, and Gyu-Chul Yi

Citation: Applied Physics Letters 87, 033101 (2005); doi: 10.1063/1.1990247

View online: http://dx.doi.org/10.1063/1.1990247

View Table of Contents: http://scitation.aip.org/content/aip/journal/apl/87/3?ver=pdfcov

Published by the AIP Publishing

\section{Articles you may be interested in}

Optical absorption of Mg-doped layers and InGaN quantum wells on c-plane and semipolar GaN structures J. Appl. Phys. 113, 203108 (2013); 10.1063/1.4806997

Photoexcitation screening of the built-in electric field in $\mathrm{ZnO}$ single quantum wells

Appl. Phys. Lett. 93, 121907 (2008); 10.1063/1.2981523

Structural and optical properties of $\mathrm{Zn} \mathrm{O} / \mathrm{Mg} 0.1 \mathrm{Zn} 0.9 \mathrm{O}$ multiple quantum wells grown on $\mathrm{ZnO}$ substrates Appl. Phys. Lett. 90, 211909 (2007); 10.1063/1.2742574

High-quality, melt-grown $\mathrm{ZnO}$ single crystals

J. Appl. Phys. 95, 4802 (2004); 10.1063/1.1691186

Polarized photoreflectance spectra of excitonic polaritons in a $\mathrm{ZnO}$ single crystal

J. Appl. Phys. 93, 756 (2003); 10.1063/1.1527707

You don't

still use this

cell phone

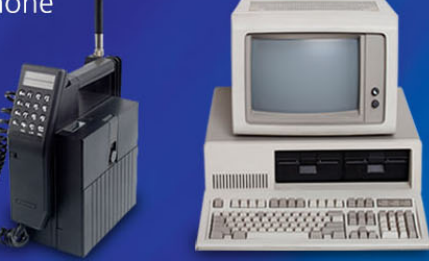

Why are you

still using an

AFM designed in the 80 's?
It is time to upgrade your AFM Minimum $\$ 20,000$ trade-in discount for purchases before August 31st

Asylum Research is today's technology leader in AFM

dropmyoldAFM@oxinst.com

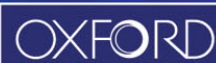




\title{
Near-field measurement of spectral anisotropy and optical absorption of isolated $\mathrm{ZnO}$ nanorod single-quantum-well structures
}

\author{
Takashi Yatsui ${ }^{\text {a) }}$ and Motoichi Ohtsu ${ }^{\text {b) }}$ \\ Solution-Oriented Research for Science and Technology, Japan Science and Technology Agency, Machida, \\ Tokyo, 194-0004 Japan \\ Jinkyoung Yoo, Sung Jin An, and Gyu-Chul Yi \\ National CRI Center for Semiconductor Nanorods and Department of Materials Science and Engineering, \\ Pohang University of Science and Technology (POSTECH), San 31 Hyoja-dong, Pohang, Gyeongbuk \\ 790-784, Korea
}

(Received 3 February 2005; accepted 10 June 2005; published online 11 July 2005)

\begin{abstract}
We report low-temperature near-field spectroscopy of isolated $\mathrm{ZnO} / \mathrm{ZnMgO}$ single-quantum-well structures (SQWs) on the end of $\mathrm{ZnO}$ nanorod to define their potential for nanophotonics. First, absorption spectra of isolated $\mathrm{ZnO} / \mathrm{ZnMgO}$ nanorod SQWs with the Stokes shift as small as $3 \mathrm{meV}$ and very sharp photoluminescent peaks indicate that the nanorod SQWs are of very high optical quality. Furthermore, we performed polarization spectroscopy of isolated $\mathrm{ZnO} \mathrm{SQWs}$, and observed valence-band anisotropy of $\mathrm{ZnO}$ SQWs in photoluminescence spectra directly. Since the exciton in a quantum structure is an ideal two-level system with long coherence times, our results provide criteria for designing nanophotonic devices. (C) 2005 American Institute of Physics.
\end{abstract}

[DOI: $10.1063 / 1.1990247]$

$\mathrm{ZnO}$ nanocrystallites are a promising material for realizing nanometer-scale photonic devices, ${ }^{1}$ i.e., nanophotonic devices, at room temperature, owing to their large exciton binding energy ${ }^{2-4}$ and large oscillator strength. ${ }^{5}$ Furthermore, the recent demonstration of semiconductor nanorod quantumwell structures enables us to fabricate nanometer-scale electronic and photonic devices on single nanorods. ${ }^{6-9}$ Recently, $\mathrm{ZnO} / \mathrm{ZnMgO}$ nanorod heterostructures were fabricated and the quantum confinement effect even from the singlequantum-well structures (SQWs) was observed. ${ }^{10}$ Near-field spectroscopy has made a remarkable contribution to investigations of the optical properties in nanocrystallite, ${ }^{11}$ and has resulted in the observation of nanometer-scale optical images, such as the local density of exciton states. ${ }^{12}$ However, reports on semiconductor quantum structure are limited to naturally formed quantum dots (QDs). ${ }^{12-14}$ Here we report low-temperature near-field spectroscopy of artificially fabricated $\mathrm{ZnO}$ SQWs on the end of a $\mathrm{ZnO}$ nanorod.

$\mathrm{ZnO} / \mathrm{ZnMgO}$ SQWs were fabricated on the ends of $\mathrm{ZnO}$ stems with a mean diameter of $40 \mathrm{~nm}$ and a length of $1 \mu \mathrm{m}$ using catalyst-free metalorganic vapor phase epitxy, in which the $\mathrm{ZnO}$ nanorods were grown vertically from a sapphire (0001) substrate in the $c$ orientation. ${ }^{10,15}$ The $\mathrm{Mg}$ concentration in the $\mathrm{ZnMgO}$ layers averaged 20 at. \%. Two samples were prepared for this study: their $\mathrm{ZnO}$ well layer thickness $L_{w}$, were 2.5 and $3.75 \mathrm{~nm}$, while the thicknesses of the $\mathrm{Zn}$ $\mathrm{MgO}$ bottom and top barrier layers in the SQWs were fixed at 60 and $18 \mathrm{~nm}$, respectively. After growing the $\mathrm{ZnO} / \mathrm{ZnMgO}$ nanorod SQWs, they were dispersed so that they were laid down on a flat sapphire substrate to isolate them from each other [Fig. 1(a)].

The far-field photoluminescence (PL) spectra were obtained using a $\mathrm{He}-\mathrm{Cd}$ laser $(\lambda=325 \mathrm{~nm})$ before dispersion of the $\mathrm{ZnO} / \mathrm{ZnMgO}$ nanorod SQWs. The emission signal was

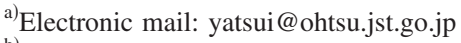

${ }^{b)}$ Also at: School of Engineering, The University of Tokyo, Bunkyo-ku, Tokyo, 113-8656 Japan.
}

collected with the acromatic lens $(f=50 \mathrm{~mm})$. To confirm that the optical qualities of individual $\mathrm{ZnO} / \mathrm{ZnMgO} \mathrm{SQWs}$ were sufficiently high, we used a collection-mode near-field optical microscope (NOM) using a $\mathrm{He}-\mathrm{Cd}$ laser $(\lambda$ $=325 \mathrm{~nm}$ ) for excitation, and a UV fiber probe with an aperture diameter of $30 \mathrm{~nm}$. The excitation source was focused on a nanorod sample laid on the substrate with a spot size approximately $100 \mu \mathrm{m}$ in diameter. The PL signal was collected with the fiber probe, and detected using a cooled charge coupled device through a monochromator. The fiber probe was kept in close proximity to the sample surface $(\sim 5 \mathrm{~nm})$ using the shear-force feedback technique. The polarization of the incident light was controlled with a $\lambda / 2$

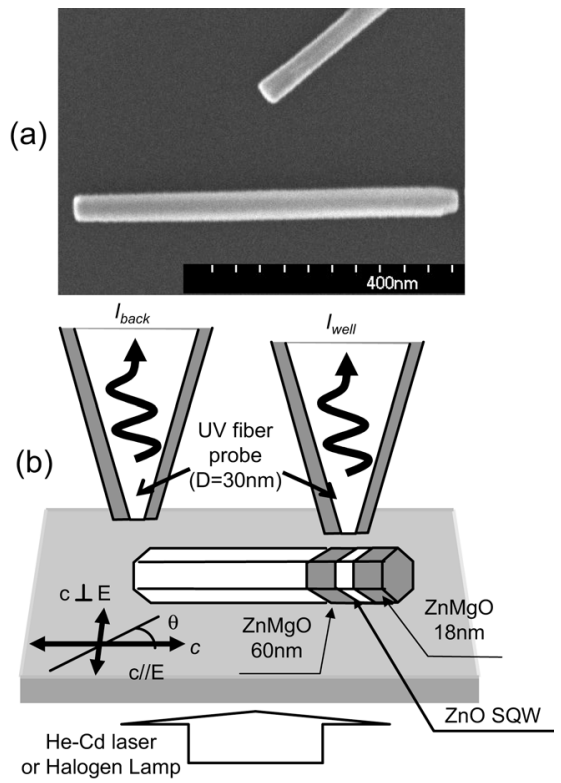

FIG. 1. Schematic of experimental setup for near-field PL spectroscopy. (a) Scanning electron micrographic image of the dispersed $\mathrm{ZnO} / \mathrm{ZnMgO}$ SQWs. (b) Schematic of $\mathrm{ZnO} / \mathrm{ZnMgO}$ SQWs on the ends of $\mathrm{ZnO}$ nanorods. $c: c$ axis of the $\mathrm{ZnO}$ stem. $\theta$ : angle between the $\mathrm{ZnO}$ stem and the direction of incident light polarization. 


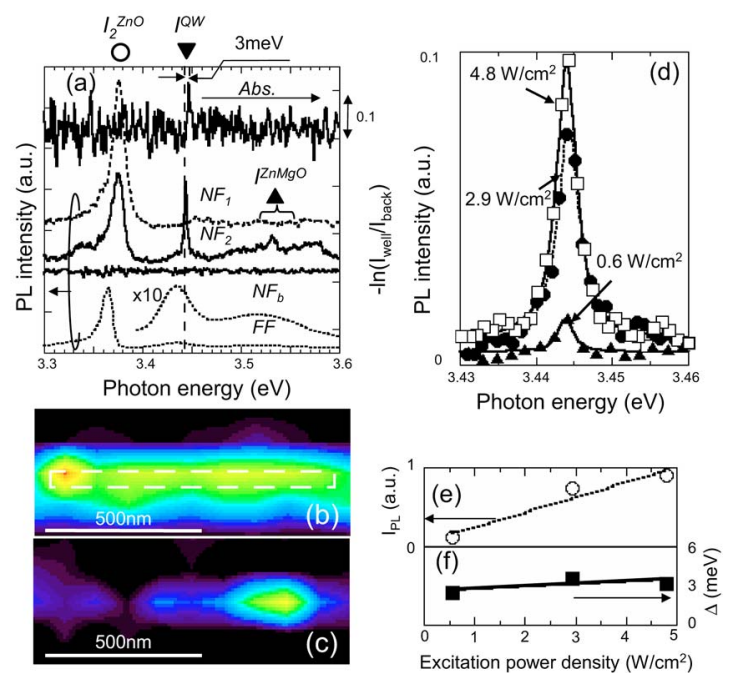

FIG. 2. (Color online) Near-field PL and absorption spectroscopy of isolated $\mathrm{ZnO}$ SQWs $\left(L_{w}=3.75 \mathrm{~nm}\right)$ at $15 \mathrm{~K}$. (a) $\mathrm{NF}_{1}, \mathrm{NF}_{2}$ : near-field PL spectra. $\mathrm{NF}_{b}$ : background noise. Abs.: near-field absorption spectrum. FF: far-field $\mathrm{PL}$ spectrum of vertically aligned $\mathrm{ZnO}$ SQWs $\left(L_{w}=3.75 \mathrm{~nm}\right)$. NOM images of isolated $\mathrm{ZnO}$ SQWs obtained at (b) 3.375 and (c) $3.444 \mathrm{eV}$. The rectangle shown in dashed white lines indicates the position of the $\mathrm{ZnO}$ stem. (d) Near-field PL spectra of isolated $\mathrm{ZnO}$ SQWs at excitation densities ranging from 0.6 to $4.8 \mathrm{~W} / \mathrm{cm}^{2}$. The integrated PL intensity $I_{\mathrm{PL}}$ (e) and homogeneous linewidths $\Delta$ (f) as a function of the excitation power density.

wave plate. In contrast to the naturally formed QD structure (a high monolayer island formed in a narrow quantum well), the barrier and cap layers laid on the substrate allowed the probe tip access to the PL source, which reduced carrier diffusion in the $\mathrm{ZnO}$ SQWs and the subsequent linewidth broadening, thereby achieving a high spatial and spectral resolution. In addition to the PL measurements, absorption spectra were obtained using a halogen lamp, where the absorption was defined by the ratio $I_{\text {well }} / I_{\text {back }}$ between the signal intensities transmitted through the well layer $\left(I_{\text {well }}\right)$ and substrate $\left(I_{\text {back }}, 50 \mathrm{~nm}\right.$ apart from the well layer) [Fig. 1(b)]. The absorption signal was collected with the same fiber probe with an aperture diameter of $30 \mathrm{~nm}$. Since the $\mathrm{ZnMgO}$ layers are much thicker than that of the well layer $(\sim 3 \mathrm{~nm})$, any difference in the transmission signals between $I_{\text {well }}$ and $I_{\text {back }}$ was not detected, which resulted in no detection of the absorption peak originating from the $\mathrm{Zn}$ $\mathrm{MgO}$ layers.

As a preliminary near-field spectroscopy experiment of the ZnO SQWs, we obtained near-field PL spectra of the $\mathrm{ZnO}$ SQWs with $L_{w}=3.75 \mathrm{~nm}$ [Fig. 2(a)] obtained with polarization perpendicular to the $c$ axis $\left[\theta=90^{\circ}\right.$ in Fig. 1(b)]. Two typical spectra are shown, one with a single peak at $3.375 \mathrm{eV}\left(\mathrm{NF}_{1}\right)$ and the other with several sharp peaks around $3.375,3.444$, and $3.530 \mathrm{eV}\left(\mathrm{NF}_{2}\right)$, while $\mathrm{NF}_{b}$ is a background spectra [Fig. 2(a)]. Several conclusions can be drawn from these spectral profiles. First, comparison with the far-field PL spectrum [FF: dashed curve in Fig. 2(a)] showed that the emission peak $I_{2}^{\mathrm{ZnO}}$ at $3.375 \mathrm{eV}$ was suppressed, and $I^{\mathrm{QW}}(3.444 \mathrm{eV})$ and $I^{\mathrm{ZnMgO}}(3.530 \mathrm{eV})$ were enhanced in $\mathrm{NF}_{2}$, indicating that peaks $I_{2}^{\mathrm{ZnO}}$ and $I^{\mathrm{ZnMgO}}$ originated from the $\mathrm{ZnO}$ stem and $\mathrm{ZnMgO}$ layers, respectively. Second, since the peak position of $I^{\mathrm{QW}}$ was consistent with the theoretical prediction $(3.430 \mathrm{eV})$ using the finite squarewell potential of the quantum confinement effect in the $\mathrm{ZnO}$ well layer for $L_{w}=3.75 \mathrm{~nm}$, we concluded that peak $I^{\mathrm{QW}}$ originated from the $\mathrm{ZnO} \mathrm{SQWs}$. The theoretical calculation

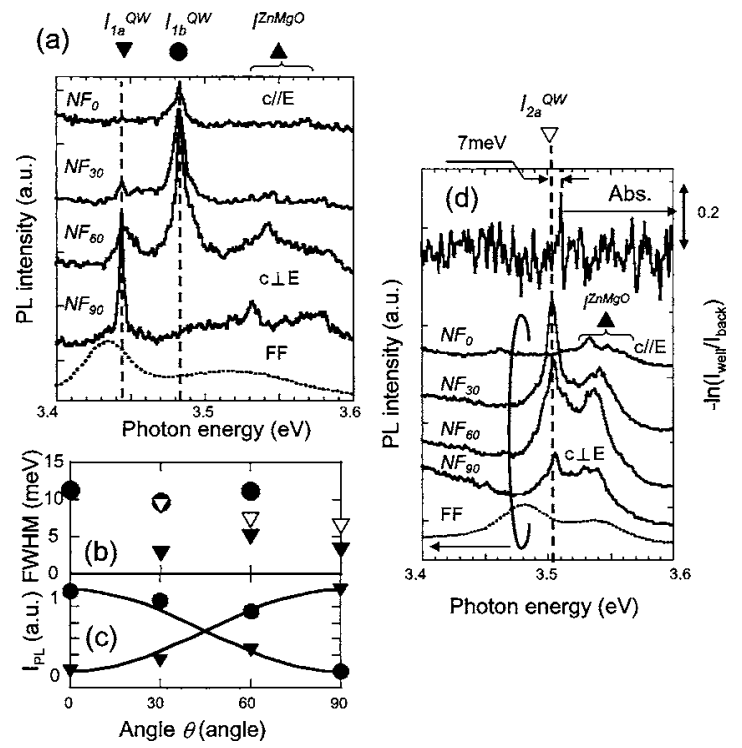

FIG. 3. Polarization dependence of near-field PL spectra of isolated $\mathrm{ZnO}$ SQWs obtained at $15 \mathrm{~K}$. (a) $\mathrm{NF}_{\theta}, \mathrm{FF}$ : near-field and far-field PL spectra of isolated $\mathrm{ZnO}$ SQWs $\left(L_{w}=3.75 \mathrm{~nm}\right)$ for $\theta=0^{\circ}, 30^{\circ}, 60^{\circ}$, and $90^{\circ}$. (b) Solid triangles and circles are the polarization dependence of the linewidth of $I_{1 a}^{\mathrm{QW}}$ and $I_{1 b}^{\mathrm{QW}}$, respectively in (a). Open triangles are the polarization dependence of linewidth of $I_{2 a}^{\mathrm{QW}}$ in (d). (c) Solid triangles and circles are the integrated $\mathrm{PL}$ intensities $\left(I_{\mathrm{PL}}\right)$ of $I_{1 a}^{\mathrm{QW}}$ and $I_{1 b}^{\mathrm{QW}}$, respectively, normalized to the total PL intensities $\left(I_{1 a}^{\mathrm{QW}}+I_{1 b}^{\mathrm{QW}}\right)$. (d) $\mathrm{NF}_{\theta}$, FF: near-field and far-field PL spectra, respectively, of isolated $\mathrm{ZnO}$ SQWs $\left(L_{w}=2.5 \mathrm{~nm}\right)$. Abs.: absorption spectrum.

used $0.28 m_{0}$ and $1.8 m_{0}$ as the effective masses of an electron and hole in $\mathrm{ZnO}$, respectively, at a ratio of conduction- and valence-band offsets $\left(\Delta E_{c} / \Delta E_{v}\right)$ of 9 , and a band-gap offset $\left(\Delta E_{g}\right)$ of $250 \mathrm{meV} .^{10}$ The spatial distributions of the nearfield PL intensity of peaks $I_{2}^{\mathrm{ZnO}}$ and $I^{\mathrm{QW}}$ [Figs. 2(b) and 2(c)] supported the postulate that the blueshifted emission was confined to the end of the $\mathrm{ZnO}$ stem. Third, the spectral width $(3 \mathrm{meV})$ of peak $I^{\mathrm{QW}}$ was much narrower than those of the far-field PL spectra $(40 \mathrm{meV})$. To estimate the homogeneous linewidth of isolated $\mathrm{ZnO} \mathrm{SQWs}$, we observed the power dependence of the near-field PL spectra [Fig. 2(d)] by varying the excitation power densities from 0.6 to $4.8 \mathrm{~W} / \mathrm{cm}^{2}$. The shape of each spectrum was fitted using the Lorentzian function indicated by the solid curve. Figures 2(e) and 2(f) show the integrated PL intensity $\left(I_{\mathrm{PL}}\right)$ and linewidth $(\Delta)$ of the fitted Lorentzian, which increased linearly and remained constant around $3 \mathrm{meV}$, respectively. These results indicate that emission peak $I^{\mathrm{QW}}$ represented the emission from a single-exciton state in $\mathrm{ZnO} \mathrm{SQWs}$ and that the linewidth was governed by the homogeneous broadening. Fourth, the Stokes shift of $3 \mathrm{meV}$ [Fig. 2(a)] was much smaller than the reported value $(50 \mathrm{meV})$ in $\mathrm{ZnO} / \mathrm{ZnMgO}$ superlattices. ${ }^{16,17}$ The small Stokes shift may result from the decreased piezoelectric polarization effect by the fully relaxed strain for the $\mathrm{ZnO} / \mathrm{ZnMgO}$ nanorod quantum structures in contrast to the two-dimensional (2D) $\mathrm{ZnO} / \mathrm{ZnMgO}$ heteroepitaxial multiple layers are strongly supported by the theoretical calculation on the double barrier $\ln A s / \operatorname{lnP}$ nanorod heterostructures. ${ }^{18}$

Based on these experiments, a major investigation of the optical properties of isolated $\mathrm{ZnO} \mathrm{SQWs}$ was performed by analyzing the polarization-dependent PL spectrum of isolated ZnO SQWs $\left(L_{w}=3.75 \mathrm{~nm}\right)$. As shown in Fig. $3(\mathrm{a}), \mathrm{NF}_{0}$ is a near-field PL spectrum obtained with parallel polarization with respect to the $c$ axis, $\theta=0^{\circ}$, and this exhibits a new peak 
$I_{1 b}^{\mathrm{QW}}$ at $3.483 \mathrm{eV}$, which is out of peak in the far-field spectrum $(3.435 \mathrm{eV} \pm 20 \mathrm{meV})$. Peak $I_{1 a}^{\mathrm{QW}}$ is the same as $I^{\mathrm{QW}}$ in Fig. 2(a).

As the $\mathrm{ZnO}$ has valence-band anisotropy owing to the wurtzite crystal structure, the operator corresponds to the $\Gamma_{5}\left(\Gamma_{1}\right)$ representation when the electric vector $\boldsymbol{E}$ of the incident light is perpendicular (parallel) to the crystalline $c$ axis, respectively. By considering the energy difference between $\Gamma_{5}$ and $\Gamma_{1}$ in the center of the zone around $40 \mathrm{meV}$ for bulk material, ${ }^{5,19,20}$ and the direction of the incident light polarization with respect to the $c$ axis, emission peaks $I_{1 a}^{\mathrm{QW}}$ and $I_{1 b}^{\mathrm{QW}}$ in Fig. 3(a) are allowed for the exciton from $\Gamma_{5}$ and $\Gamma_{1}$, respectively. This observation of a $\Gamma_{1}$ exciton in a PL spectrum originates from the enhancement of the exciton binding energy owing to the quantum confinement effect ${ }^{4}$ because the exciton binding energy of the emission from $\Gamma_{1}(50-56 \mathrm{meV}$ ) (see Refs. 20 and 21) is comparable to that from $\Gamma_{5}(60 \mathrm{meV})$. Furthermore, in contrast to the bulk $\mathrm{ZnO}$ film, our sample configuration using laid $\mathrm{ZnO}$ nanorod SQWs has realized $\pi$ polarization $\left(\theta=0^{\circ}\right)$, allowing the detection of the emission from the $\Gamma_{1}$ exciton. The homogeneous linewidth of emission peak $I_{1 a}^{\mathrm{QW}}\left(\Gamma_{5}\right)$ is in the range $3-5 \mathrm{meV}$, while that of $I_{1 b}^{\mathrm{QW}}\left(\Gamma_{1}\right)$ is $9-11 \mathrm{meV}$ [Fig. 3(b)]. This difference is attributed to the degeneracy of the transition of the $\Gamma_{1}$ exciton with continuum and to the contribution of the residual strain field, and results in sensitive dependence of the $\Gamma_{1}$ exciton on the strain, as reported in the $\mathrm{GaN}^{22}$ The solid triangles and circles in Fig. 3(c) shows the respective normalized integrated PL intensity at $I_{1 a}^{\mathrm{QW}}$ and $I_{1 b}^{\mathrm{QW}}$, respectively, which are in good agreement with the sinesquared and cosine-squared functions represented by the solid curves. These results indicate that emission peaks $I_{1 a}^{\mathrm{QW}}$ and $I_{1 b}^{\mathrm{QW}}$ originate from unidirectional transition dipoles that are orthogonal to each other.

To study the linewidth broadening mechanism, Fig. 3(d) shows the polarization-dependent near-field PL spectra $\left(\mathrm{NF}_{0}-\mathrm{NF}_{90}\right)$ and absorption spectrum obtained for isolated $\mathrm{ZnO}$ SQWs with a thinner well layer $\left(L_{w}=2.5 \mathrm{~nm}\right)$. In $\mathrm{NF}_{0}-\mathrm{NF}_{90}$, the emission peaks $I^{\mathrm{ZnMgO}}$ around $3.535 \mathrm{eV}$ originate from the $\mathrm{ZnMgO}$ layers. Emission peak $I_{2 a}^{\mathrm{QW}}$ originates from the $\Gamma_{5}$ exciton in the SQWs, as was the case for $I_{1 a}^{\mathrm{QW}}$ in Fig. 3(a), since the position of peak $I_{2 a}^{\mathrm{QW}}(3.503 \mathrm{eV})$ is comparable to that of the dominant peak in the far-field PL spectra $(3.480 \mathrm{eV})$ and the theoretical prediction $(3.455 \mathrm{eV})$ using the finite square-well potential of the quantum confinement effect in the $\mathrm{ZnO}$ well layer. In comparison to $\mathrm{ZnO}$ SQWs with $L_{w}=3.75 \mathrm{~nm}$, however, emission peak $I_{2 a}^{\mathrm{QW}}$ had a broader linewidth $(7-10 \mathrm{meV})$, which is attributed to the shorter exciton dephasing time. In the nanocrystallite where the excitons are quantized, the linewidth should be determined by the exciton dephasing time. Such dephasing arises from the collisions of the excitons at the irregular surface, so that the linewidth is $d^{-2}$ ( $d$ is the effective size of the quantum structure). ${ }^{23}$ The observed well-width dependence of the spectral linewidth, $3.75^{-2} / 2.5^{-2} \sim 3 / 7$, and the Stokes shift of $7 \mathrm{meV}$ [see Fig. 3(c)] larger than that for $L_{w}=3.75 \mathrm{~nm}$ $(3 \mathrm{meV})$ are supported by this dephasing mechanism quantitatively. Although emission peak $I_{2 a}^{\mathrm{QW}}$ was suppressed for $\theta$ $=0^{\circ}$, no peaks corresponding to the $\Gamma_{1}$ exciton in SQWs were detected owing to the reduction of the exciton binding energy, since the peak energy of $\Gamma_{1}$ for the ZnO SQWs with
$L_{w}=2.5 \mathrm{~nm}$ is comparable with that of $\mathrm{ZnMgO}$.

The results shown here provide criteria for realizing nanophotonic devices using a two-level system. ${ }^{24,25}$ As a representative device, a nanophotonic switch can be realized by controlling the dipole forbidden optical energy transfer among resonant energy levels in nanometer-scale QD via an optical near field. ${ }^{26}$ Since the switching dynamics was already confirmed using $\mathrm{CuCl}$ quantum cubes, ${ }^{26}$ the success of near-field PL and absorption measurement of isolated SQWs described above is a promising step toward designing a nanophotonic switch and related devices.

The authors are grateful to Drs. I. Banno (Yamanashi University) and S. Sangu (Ricoh Company, Ltd.) for many fruitful discussions. The work at POSTECH was supported by the National Creative Research Initiative Project, Korea and the AOARD 04-49 (Quotation No. FA5209-04-T0254).

${ }^{1}$ M. Ohtsu, K. Kobayashi, T. Kawazoe, S. Sangu, and T. Yatsui, IEEE J. Sel. Top. Quantum Electron. 8, 839 (2002).

${ }^{2}$ A. Ohtomo, K. Tamura, M. Kawasaki, T. Makino, Y. Segawa, Z. K. Tang, G. K. L. Wong, Y. Matsumoto, and H. Koinuma, Appl. Phys. Lett. 77, 2204 (2000).

${ }^{3}$ M. H. Huang, S. Mao, H. Feick, H. Yan, Y. Wu, H. Kind, E. Weber, R. Russo, and P. Yang, Science 292, 1897 (2001).

${ }^{4}$ H. D. Sun, T. Makino, Y. Segawa, M. Kawasaki, A. Ohtomo, K. Tamura, and H. Koinuma, J. Appl. Phys. 91, 1993 (2002).

${ }^{5}$ D. C. Reynolds, D. C. Look, B. Jogai, C. W. Litton, G. Cantwell, and W. C. Harsch, Phys. Rev. B 60, 2340 (1999).

${ }^{6}$ Y. Wu, R. Fan, and P. Yang, Nano Lett. 2, 83 (2002).

${ }^{7}$ M. T. Björk, B. J. Ohlsson, C. Thelander, A. I. Persson, K. Deppert, L. R. Wallenberg, and L. Samuelson, Appl. Phys. Lett. 81, 4458 (2003).

${ }^{8}$ M. S. Gudiksen, L. J. Lauhon, J. Wang, D. C. Smith, and C. M. Lieber, Nature (London) 415, 617 (2002).

${ }^{9}$ W. I. Park, G.-C. Yi, M. Kim, and S. J. Pennycook, Adv. Mater. (Weinheim, Ger.) 15, 526 (2003).

${ }^{10}$ W. I. Park, S. J. An, J. L. Yang, G.-C. Yi, S. Hong, T. Joo, and M. Kim, J. Phys. Chem. B 108, 15457 (2004).

${ }^{11}$ K. Matsuda, T. Saiki, S. Nomura, M. Mihara, and Y. Aoyagi, Appl. Phys. Lett. 81, 2291 (2002).

${ }^{12}$ K. Matsuda, T. Saiki, S. Nomura, M. Mihara, Y. Aoyagi, S. Nair, and T. Takagahara, Phys. Rev. Lett. 91, 177401 (2003).

${ }^{13}$ J. R. Guest, T. H. Stievater, G. Chen, E. A. Tabak, B. G. Orr, D. G. Steel, D. Gammon, and D. S. Katzer, Science 293, 2224 (2001).

${ }^{14}$ T. Guenther, C. Lienau, T. Elsaesser, M. Glanemann, V. M. Axt, T. Kuhn, S. Eshlaghi, and A. D. Wieck, Phys. Rev. Lett. 89, 057401 (2002).

${ }^{15}$ W. I. Park, D. H. Kim, S.-W. Jung, and G.-C. Yi, Appl. Phys. Lett. 80, 4232 (2002).

${ }^{16}$ A. Ohtomo, M. Kawasaki, I. Ohkubo, H. Koinuma, T. Yasuda, and Y. Segawa, Appl. Phys. Lett. 75, 980 (1999).

${ }^{17}$ T. Makino, A. Ohtomo, C. H. Chia, Y. Segawa, H. Koinuma, and M. Kawasaki, Physica E (Amsterdam) 21, 671 (2004).

${ }^{18}$ M. Zervos and L.-F. Feiner, J. Appl. Phys. 95, 281 (2004).

${ }^{19}$ M. Zamfirescu, A. Kavokin, B. Gil, G. Malpuech, and M. Kaliteevski, Phys. Rev. B 65, 161205 (2002).

${ }^{20}$ S. F. Chichibu, T. Sota, G. Cantwell, D. B. Eason, and C. W. Litton, J. Appl. Phys. 93, 756 (2003).

${ }^{21}$ D. C. Reynolds, C. W. Litton, D. C. Look, J. E. Hoelscher, B. Claflin, T. C. Collins, J. Nause, and B. Nemeth, J. Appl. Phys. 95, 4802 (2004).

${ }^{22}$ M. Tchounkeu, O. Briot, B. Gil, J. P. Alexis, and R.-L. Aulombard, J. Appl. Phys. 80, 5352 (1996).

${ }^{23}$ T. Wamura, Y. Masumoto, and T. Kawamura, Appl. Phys. Lett. 59, 1758 (1991).

${ }^{24}$ A. Zrenner, E. Beham, S. Stufler, F. Findeis, M. Bichler, and G. Abstreiter, Nature (London) 418, 612 (2002).

${ }^{25}$ Z. Yuan, B. E. Kardynal, R. M. Stevenson, A. J. Shields, C. J. Lobo, K. Cooper, N. S. Beattie, D. A. Ritchie, and M. Pepper, Science 295, 102 (2002).

${ }^{26}$ T. Kawazoe, K. Kobayashi, S. Sangu, and M. Ohtsu, Appl. Phys. Lett. 82, 2957 (2003). 